BMJ Open

Diabetes

Research

\& Care

\section{RUNX1 can mediate the glucose and O-GlcNAc-driven proliferation and migration of human retinal microvascular endothelial cells}

To cite: Xing X, Wang H, Niu T, et al. RUNX1 can mediate the glucose and

0-GIcNAc-driven proliferation and migration of human retinal microvascular endothelial cells. BMJ Open Diab Res Care 2021;9:e001898. doi:10.1136/ bmjdrc-2020-001898

Received 10 September 2020 Accepted 17 July 2021
Check for updates

C Author(s) (or their employer(s)) 2021. Re-use permitted under CC BY-NC. No commercial re-use. See rights and permissions. Published by BMJ.

${ }^{1}$ Department of Ophthalmology, Shanghai General Hospital, Shanghai Jiao Tong University School of Medicine, Shanghai, China

${ }^{2}$ National Clinical Research Center for Eye Diseases, Shanghai, China

${ }^{3}$ Shanghai Key Laboratory of Ocular Fundus Diseases, Shanghai, China

${ }^{4}$ Shanghai Engineering Center for Visual Science and Photomedicine, Shanghai, China

${ }^{5}$ Shanghai Engineering Center for Precise Diagnosis and Treatment of Eye Diseases, Shanghai, China

Correspondence to

Dr Kun Liu;

drliukun@sjtu.edu.cn

\section{ABSTRACT}

Introduction This study aims to determine whether high glucose condition and dynamic 0 -linked $\mathrm{N}$ acetylglucosamine (0-GlcNAc) modification can promote the proliferation and migration of human retinal microvascular endothelial cells (HRMECs) and whether Runt-related transcription factor 1 (RUNX1) could mediate the glucose and 0-GICNAc-driven proliferation and migration of HRMECs.

Research design and methods Western blot analysis was used to detect the 0-GlcNAc modification level and RUNX1 level in cells and retina tissues, cell growth was studied by cell counting kit-8 assay, cell proliferation was detected by immunofluorescence staining. Then, cell migration and tube formation were investigated by scratch-wound assay, Transwell assay, and tube-forming assay. The changes of retinal structure were detected by H\&E staining. The 0-GICNAc modification of RUNX1 was detected by immunoprecipitation.

Results High glucose increases pan-cellular 0-GlcNAc modification and the proliferation and migration of HRMECs. Hence, 0-GIcNAc modification is critical for the proliferation and migration of HRMECs. RUNX1 mediates the glucose and 0-GlcNAc-driven proliferation and migration in HRMECs. RUNX1 can be modified by 0 GICNAc, and that the modification is enhanced in a high glucose environment.

Conclusions The present study reveals that high glucose condition directly affects retinal endothelial cells (EC) function, and 0-GIcNAc modification is critical for the proliferation and migration of HRMECs, RUNX1 may take part in this mechanism, and maybe the function of RUNX1 is related to its 0-GICNAc modification level, which provides a new perspective for studying the mechanism of RUNX1 in diabetic retinopathy.

\section{INTRODUCTION}

Diabetic retinopathy (DR) is one of the complications caused by diabetes, and is the leading cause of blindness of the working-age population. ${ }^{1}$ DR can be divided into two stages: non-proliferative diabetic retinopathy and proliferative diabetic retinopathy (PDR). Neovascularization is a pathological feature of PDR. ${ }^{2}$ In recent days, a meta-analysis study

\section{Significance of this study}

What is already known about this subject?

- High glucose can improve the level of 0 -linked $\mathrm{N}$ acetylglucosamine (0-GIcNAc) modification of retina vascular endothelial cells $(E C)$.

- High glucose regulates Runt-related transcription factor 1 (RUNX1) and implicates RUNX1 in aberrant retinal angiogenesis.

What are the new findings?

- High glucose condition directly affects retinal EC function.

- 0 -GICNAc modification is critical for the proliferation and migration of human retinal microvascular endothelial cells (HRMECs).

- 0 -GICNAc can modify RUNX1, and the modification is enhanced in a high glucose environment, and maybe the function of RUNX1 is related to its $0-$ GICNAC modification level.

How might these results change the focus of research or clinical practice?

- Controlling the increase of 0 -GIcNAc modification may be beneficial for the occurrence and development of diabetic retinopathy (DR).

- This research provides a new direction to study the mechanism of RUNX1 in DR; that is, we can explore the role of RUNX1 from the perspective of protein post-translational modification.

that included 22896 patients with diabetes revealed that for patients with diabetes, the prevalence of patients with DR was $34.6 \%$. Furthermore, among these patients, the prevalence of PDR was $6.96 \%$, while the prevalence of diabetic macular edema was $6.81 \%$. $^{3}$ The pathological changes of DR is retinal capillary endothelial damage, which includes selective loss of retinal capillary pericytes, a thickened basement membrane, occluded capillaries and vascular leakage, and these are due to the blood-retinal barrier damage. These changes lead to a wide range of retinal 
ischemia and retinal hypoxia, which eventually lead to the formation of new blood vessels. DR is a chronic disease. On the basis of hyperglycemia, multiple factors can lead to the occurrence and development of $\mathrm{DR},{ }^{45}$ such as the increased polyol pathway activity, the activation of the protein kinase $\mathrm{C}$ and hexosamine biosynthesis pathway (HBP), oxidative damage to the retina, and increased formation of advanced glycated end-products. However, the specific molecular mechanism remains not completely clear.

O-linked N-acetylglucosamine (O-GlcNAc) modification is a unique form of post-translational protein modification (PTM). Its uniqueness is embodied in its highly dynamic and revisible form of PTM. This can be regulated by two enzymes: O-GlcNAc transferase (OGT) and O-GlcNAcase (OGA). Furthermore, this uses UDPGlcNAc as a donor, under the catalysis of OGT, it connects the O-glycosidic bond to protein serine/threonine residues, and under the catalysis of OGA, it hydrolyzes O-GlcNAc from serine/threonine residues. ${ }^{6}$ The changes in blood glucose levels can influence the UDP-GlcNAc level through the HBP pathway, and affect the O-GlcNAc modification of proteins. The most important feature of diabetes is sustained hyperglycemia. Elevated blood glucose can lead to increased UDP-GlcNAc donor, and subsequently lead to increased O-GlcNAc modification of proteins. Previous studies have revealed that O-GlcNAc modification plays an important role in diabetic cardiomyopathy and diabetic nephropathy. ${ }^{7}$ Recent studies have revealed that O-GlcNAc modification has similar effects in the process of DR. ${ }^{9}$ In high glucose (HG)cultured retina endothelial cells, glycosyl transferases participate in the glycosylation process, and these glycosylation products change. ${ }^{10}$ In diabetic rat retina tissues, the O-GlcNAc modification of proteins increase. ${ }^{11}$

Neovascularization is a sign of PDR. Retinal neovascularization rupture leads to vitreous hemorrhage, and causes retinal vasoconstriction and traction retinal detachment, which are the main causes of visual impairment in patients with DR. ${ }^{12}{ }^{13}$ Neovascularization is a dynamic and balanced process, which is regulated by angiogenic factors, such as vascular endothelial growth factor (VEGF) and antiangiogenic factors, such as pigment epithelium-derived factors (PEDFs). ${ }^{14}$ Under the action of angiogenic factors, vascular endothelial cells proliferate, migrate and form the lumen, and subsequently form new blood vessels. At present, the treatment for DR is neovascularization with the intraocular injection of anti-VEGF drugs, such as lucentis, zivaflibercept and conbercept, which have achieved good therapeutic effects. However, there are some side effects with the intraocular injection of anti-VEGF drugs, such as intraocular hemorrhage, endophthalmitis, neuroretinal injury, and ischemia-reperfusion injury. ${ }^{15-18}$ Therefore, ophthalmologists are still searching for new targets and the mechanism of DR neovascularization. Previous studies have found that in high-glucose cultured retinal cells, the O-GlcNAc modification of transcription factor specificity protein 1 (Sp1) can promote the expression of VEGF. ${ }^{19}$ The upregulation of VEGF expression is the direct cause of the neovascularization of DR. ${ }^{20}$ These above findings suggest that there are some relationships between O-GlcNAc modification and the formation of retinal neovascularization.

Runt-related transcription factor 1 (RUNX1) is a member of the Runx transcription factor family, and plays an important role in the determination of cell lineage differentiation direction, the formation of normal hematopoietic cells, and stem cell proliferation. ${ }^{21}$ RUNX1 was first obtained by the Miyoshi research group in 1991 in the leukemia cell clone of patients with acute myeloid leukemia. Hence, this was named AML1. ${ }^{22}$ Previous studies have revealed that the inhibition of RUNX1 can significantly inhibit the formation of retinal neovascularization in hypoxia-induced retinal neovascularization model mice. The knockdown of RUNX1 in human retinal microvascular endothelial cells (HRMECs) can reduce the ability of cells to form tubes, ${ }^{23}$ indicating that RUNX1 has a clear role in promoting angiogenesis. However, its mechanism remains unknown.

This present study investigates whether high glucose conditions and increased O-GlcNAc modification can promote the proliferation and migration of HRMECs and whether RUNX1 takes part in this process.

\section{MATERIALS AND METHODS}

Animals

Adult male Sprague-Dawley (SD) rats (250-300g) were obtained from the Shanghai Laboratory Animal Center (Shanghai, China). Healthy male SD rats were randomly divided into two groups: wild-type (WT) group and diabetes mellitus group (DM).

\section{Streptozotocin solution preparation method}

The streptozotocin (STZ) powder was dissolved in $0.1 \mathrm{~mol} / \mathrm{L}$ of sodium citrate buffer, and a $10 \mathrm{mg} / \mathrm{mL} \mathrm{STZ}$ solution was obtained. Then, $0.22 \mu \mathrm{m}$ of bacteria filter was used for filtration and sterilization.

The STZ (Sigma-Aldrich, St. Louis, USA) was injected into the rat's abdomen $(45 \mathrm{mg} / \mathrm{kg})$. Then, the diabetes was confirmed by assaying the glucose concentration in blood, which was collected from the tail vein using a precision glucometer (Roche Diabetes Care, Mannheim, Germany) weekly after the STZ injection. Rats with a blood glucose concentration of $>16.7 \mathrm{mmol} / \mathrm{L}$ were considered diabetic. All rats were sacrificed at 6 months after STZ injection.

\section{Cells and treatments}

The HRMECs were purchased from Cell Systems (Danvers, USA). The endothelial cell medium (ECM-2) was purchased from ScienCell (Carlsbad, USA), $500 \mathrm{~mL}$ medium contains $5 \%$ fetal bovine serum (FBS) (v/v), $1 \%$ penicillin and streptomycin (v/v), and $1 \%$ endothelial cell growth supplement $(\mathrm{v} / \mathrm{v})$. 
Cells were treated with a medium containing $5.5 \mathrm{mM}$ of glucose (normal $(\mathrm{N})$ ) or $25 \mathrm{mM}$ of glucose $(\mathrm{HG})$, and incubated at $37^{\circ} \mathrm{C}$ with $5 \% \mathrm{CO}_{2}$. The OGA inhibitor Thiamet-G was purchased from Sigma-Aldrich, and the concentration used for the present experiments was 50 $\mu \mathrm{M}$. The OGT inhibitor ST045849 was purchased from TimTec (Newark, USA), and the concentration used for the present experiments was $25 \mu \mathrm{M}$.

\section{Immunoprecipitation}

The SD rat retinas and cells were lysed using western blot analysis and immunoprecipitation (IP) lysis buffer (Sangon, Shanghai, China). Then, the protein concentration was assessed by bicinchoninic acid (BCA) assay. An appropriate amount of primary antibody was added to $1000 \mu \mathrm{g}$ total protein (anti-O-GlcNAc antibody, PTM BioLabs $(4 \mu \mathrm{g})$, anti-RUNX1 antibody from Santa Cruz $(4 \mu \mathrm{g})$ and normal mouse IgG antibody from Cell Signaling Technology $(4 \mu \mathrm{g}))$. The antigen-antibody complex was slowly shaken on the rotating shaker at $4^{\circ} \mathrm{C}$ overnight. Then, $35 \mu \mathrm{L}$ of protein $\mathrm{A} / \mathrm{G}$ agarose beads (Invitrogen, Carlsbad, USA) was added to the antigenantibody complex, and slowly shaken on a rotating shaker at $4^{\circ} \mathrm{C}$ for 2 hours. Afterwards, the complex was washed for three times, the liquid was discarded, $\mathrm{ddH}_{2} \mathrm{O}$ and $2 \times$ protein loading was added to the sample, and this was heated at $100^{\circ} \mathrm{C}$ for $10 \mathrm{~min}$. Then, the supernatant was collected and western blot analysis was performed.

\section{Western blot analysis}

The SD rat retinas and cells were lysed using western blot analysis and IP lysis buffer (Sangon, Shanghai, China). Then, the protein concentration was assessed by BCA assay. Afterwards, the samples were separated on $7.5 \%$ sodium dodecyl-sulfate polyacrylamide gel electrophoresis (SDS-PAGE) (separating gel: $2.5 \mathrm{~mL}$ acrylamide, 2.5 $\mathrm{mL}$ solution $\mathrm{B}, 0.1 \mathrm{~mL} 1 \% \mathrm{SDS}(\mathrm{w} / \mathrm{v}), 4.8 \mathrm{~mL} \mathrm{ddH}_{2} \mathrm{O}, 0.1$ $\mathrm{mL} 1 \%$ ammonium persulfate (APS) (w/v), $10 \mu \mathrm{L}$ Temed, stacking gel: $650 \mu \mathrm{L}$ acrylamide, $1.25 \mathrm{~mL}$ solution $\mathrm{D}, 50$ $\mu \mathrm{L} 1 \% \operatorname{SDS}(\mathrm{w} / \mathrm{v}), 3 \mathrm{~mL} \mathrm{ddH}_{2} \mathrm{O}, 50 \mu \mathrm{L} 1 \%$ APS (w/v), $5 \mu \mathrm{L}$ Temed, solution B and solution D were purchased from Songon Biotech, China). Then, the blots were transferred onto polyvinylidene fluoride membranes at $100 \mathrm{~V}$ for $65 \mathrm{~min}$, blocked with $5 \%$ skimmed milk (w/v), and incubated with specific primary antibody at $4^{\circ} \mathrm{C}$ overnight. The antibodies used were anti-O-GlcNAc (1:1000), anti-RUNX1 (1:1000), and anti- $\beta$-actin (1:1000, Sangon, Shanghai, China).

\section{Transfection}

The transfections were performed according to instructions of RNAiMAX (Invitrogen, USA). The RUNX1 small interfering RNA (siRNA) was purchased from GenePharma (Shanghai, China).

\section{Cell counting kit-8 assay}

Cell counting kit-8 (CCK-8) assay was performed to detect cell proliferation. Different groups of HRMECs were plated at 96-well plate with $100 \mu \mathrm{L}$ medium per well.
After culture for $0,24,48$ and 72 hours, $110 \mu \mathrm{L}$ medium containing $10 \mu \mathrm{L}$ CCK-8 (Yeasen, China) was added in each well. Cells were cultured for another 4 hours in incubator and then the absorbance was measured at a wavelength of $450 \mathrm{~nm}$. Cell viability $=[\mathrm{Ae}-\mathrm{Ab}] /[\mathrm{Ac}-\mathrm{Ab}]$, where Ae is absorbance of wells with cells, CCK-8 solution, and experimental reagents; $\mathrm{Ab}$ is absorbance of wells with medium and CCK-8 solution but without cells and Ac is absorbance of wells with cells and CCK-8 solution but without experimental reagents.

\section{Immunofluorescence staining}

Cells were fixed in $4 \%$ paraformaldehyde $(\mathrm{w} / \mathrm{v})$ for $20 \mathrm{~min}$, permeabilized with $0.25 \%$ Triton X-100 (v/v) for $20 \mathrm{~min}$, blocked in $2 \% \mathrm{BSA}(\mathrm{w} / \mathrm{v})$ for $20 \mathrm{~min}$, and incubated in anti-ki67 (Cell Signaling Technology, Danvers, USA) overnight at a concentration of 1:400. Then, the liquid was discarded, the cells were incubated with the fluorescent secondary antibody (Invitrogen, Carlsbad, USA) at a concentration of 1:200. Cell counting was performed by ImageJ.

\section{Scratch-wound migration assay}

Different groups of HRMECs were plated in a 24-well plate with $500 \mu \mathrm{L}$ of serum-free medium per well. Then, one scratch was generated per well, and the wound area was measured by ImageJ software at present and after 12 hours.

\section{Transwell migration assay}

The cell migration was accessed using a Transwell filter (Becton, Dickinson and Company). The cell suspension containing $1 \times 10^{5}$ cells was seeded on the upper chamber that contained $200 \mu \mathrm{L}$ of serum-free medium, while $600 \mu \mathrm{L}$ of ECM medium which contains $20 \%$ FBS (v/v) was added to the lower chamber. After culturing for 24 hours, these cells were fixed using $4 \%$ paraformaldehyde $(\mathrm{w} / \mathrm{v})$ for $20 \mathrm{~min}$, and stained via $5 \%$ crystal violet solution (w/v) (Songon Biotech, China) for an additional $15 \mathrm{~min}$. Cell counting was performed by Image J.

\section{Tube-forming assay}

Different groups of HRMECs were plated on a 96-well plate precoated with the basement membrane extract (Trevigen, USA), $100 \mu \mathrm{L}$ of serum-free medium was add per well. Then, the cells were imaged at 24 hours after plating, and the tube formation was quantified using the Angiogenesis Analyzer plugin of ImageJ.

\section{H\&E staining}

The rat eyeball was fixed with $4 \%$ paraformaldehyde $(\mathrm{w} / \mathrm{v})$, dehydrated by gradient alcohol, embedded in paraffin, and cut into a series of $5 \mu \mathrm{m}$-thick slices. First, a slice was dewaxed by xylene, dehydrated with gradient ethanol, and stained with hematoxylin for $6 \mathrm{~min}$, followed by running water wash for $10 \mathrm{~min}$. After differentiation by $1 \%$ hydrochloric acid alcohol (v/v) for $15 \mathrm{~s}$, the slice was rinsed by a trickle of water and counterstained with eosin for $3 \mathrm{~min}$. On dehydration with gradient ethanol and 
transparency by xylene, the slice was sealed in neutral gum. The morphological changes in the retina were observed under an optical microscope.

\section{Statistical analysis}

The software used for statistical analysis is GraphPad prism 7. Statistical significance was determined using one-way analysis of variance. A p value $<0.05$ was considered statistically significant.

\section{RESULTS}

\section{Result 1: increased glucose concentration and 0-GICNAc} modification is sufficient for proliferation and migration of HRMECS

The HRMECs were exposed to $\mathrm{N}$ or HG conditions, with or without Thiamet G. First, we found that $50 \mu \mathrm{M}$ of Thiamet G could significantly increase the O-GlcNAc modification level in HRMECs. HG condition could also increase the O-GlcNAc modification level in HRMECs (figure 1A). Then we measured cell growth by CCK-8 assay. As shown in figure 1B, compared with group N, HG condition could promote cell growth. Treatment of cells with Thiamet $\mathrm{G}$ at the same glucose concentration could also promote cell growth. We used immunofluorescence staining to investigate the effects of HG condition and Thiamet $\mathrm{G}$ on cell proliferation. The trend of Ki67positive rate was consistent with the trend of cell viability between different groups (figure 1C), this means that HG condition and high level of O-GlcNAc modification can promote cell proliferation. Scratch-wound migration assay and Transwell migration assay were selected to detect the migration ability of each group. Before that, we excluded the effect of apoptosis on migration detection, there was no change in apoptosis among the groups (figure 1D). Compared with group N, the wound closure rate increased by $16 \%$ and the total number of cells passing through the membrane nearly doubled under the condition of HG. At the same glucose concentration, treatment with Thiamet $\mathrm{G}$ could further increase the closure rate and the total number of cells passing through the membrane, this suggests that HG condition and high level of O-GlcNAc modification can promote cell migration (figure 1E,F). In addition, we detected the difference of tube formation in each group. We found that HG condition and high level of O-GlcNAc modification can also promote the formation of tube (figure 1G).

\section{Result 2: 0-GIcNAc modification is critical for the proliferation} and migration of HRMECs

In order to assess the importance of O-GlcNAc modification on cell proliferation and migration, ST045849 was used to inhibit the OGT enzyme, that is, to inhibit the O-GlcNAc modification of the cells. First, we tested the inhibitory effect of ST045849. At the same glucose concentration, ST045849 could significantly inhibit the level of pan-cellular O-GlcNAc modification (figure 2A). No matter in normal glucose concentration or HG concentration, inhibition of O-GlcNAc modification can inhibit cell growth (figure 2B), and the decrease of Ki67-positive nuclei also indicates that inhibiting O-GlcNAc modification can inhibit the proliferation of cells (figure 2C). In this part of the experiment, we still choose scratch-wound migration assay and Transwell migration assay test to detect cell migration. Before that, we also excluded the effect of apoptosis on migration detection using western blot analysis, there was no change in apoptosis among the groups (figure 2D). In HRMECs, $25 \mu \mathrm{M}$ of ST045849 could effectively reduce wound healing area and total number of transmembrane cells (figure 2E,F), which means that the level of O-GlcNAc modification is very important for cell migration. Similarly, we examined the effect of ST045849 on tube formation. We found that ST045849 could significantly inhibit the formation of tube at the same glucose concentration (figure 2G) .

\section{Result 3: RUNX1 mediates the glucose and 0-GIcNAc-driven} proliferation and migration of HRIMECs

Previous studies have revealed that the inhibition of RUNX1 can significantly inhibit the formation of retinal neovascularization in hypoxia-induced retinal neovascularization mouse model. The knockdown of RUNX1 in HRMECs can reduce the ability of cells to form tubes, ${ }^{23}$ indicating that RUNX1 has a clear role in promoting angiogenesis, but its mechanism remains unknown. In order to evaluate the role of RUNX1 in the HG and O-GlcNAcmediated induction of cell proliferation and migration, HRMECs depleted of RUNX1 by siRNA were used. The control cells expressed a scramble siRNA sequence. The RUNX1 knockdown was verified by western blot analysis prior to the experiments. siRUNX1 can effectively knockdown RUNX1 protein in cells at normal glucose concentration or HG concentration, whether using Thiamet $G$ or not, and siRUNX1 did not significantly interfere with the pan-cellular O-GlcNAc modification, compared with cells expressed a scramble siRNA sequence (figure 3A). siRUNX1 significantly slowed down cell growth, whether caused by HG or high O-GlcNAc modification level (figure 3B). HRMECs transfected with RUNX1 siRNA had fewer Ki67-positive cells compared with cells transfected with a scramble siRNA sequence 24 hours after transfection, indicating RUNX1 also contributes to HRMECs proliferation, whether it is caused by $\mathrm{HG}$ or high level of O-GlcNAc modification (figure 3C). To investigate the potential pro-angiogenic effect of RUNX1, scratch healing ability, penetration ability, and tube-forming ability were tested. Of course, before that, we also detected the apoptosis that may affect the migration and tube-formation abilities. The results showed that there was no significant difference in apoptosis among the groups (figure 3D). As shown in figure 3E,F, siRUNX1 can significantly slow down wound healing and reduce the total number of cells passing through the membrane caused by HG or high level of O-GlcNAc modification, indicating that the presence of RUNX1 is very important for cell migration. Figure $3 \mathrm{G}$ shows that knockdown of 
A

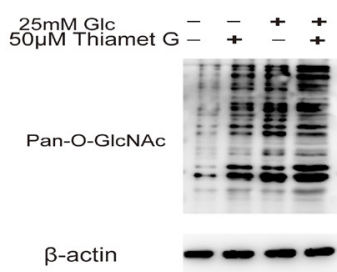

C
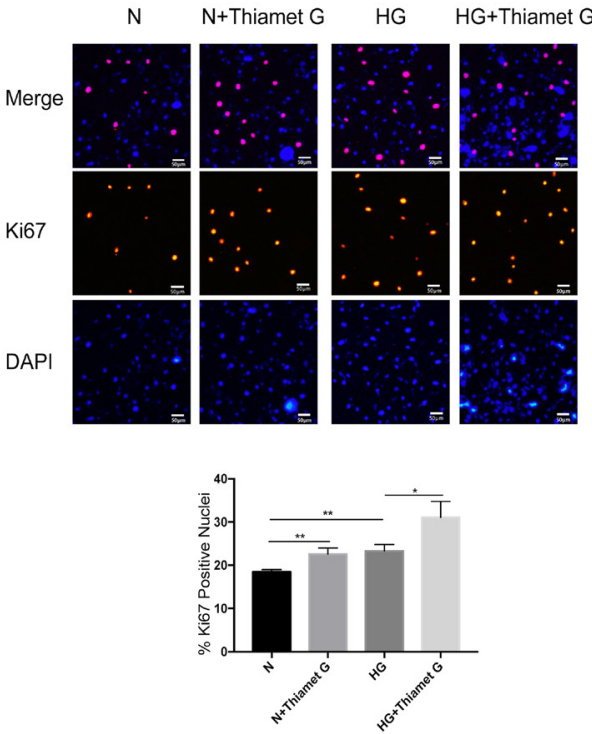

$\mathrm{E}$
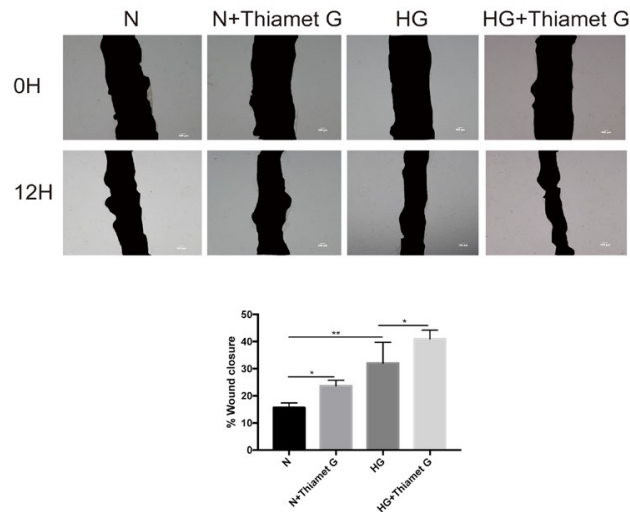

B

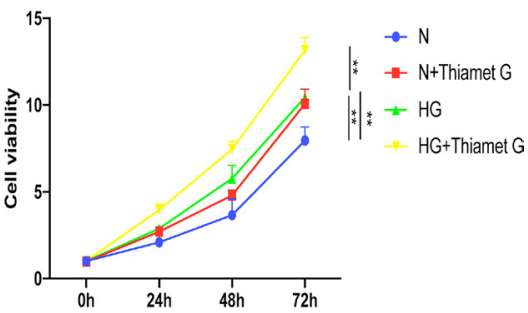

D

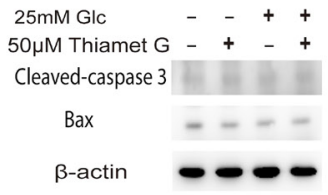

$\mathrm{F}$
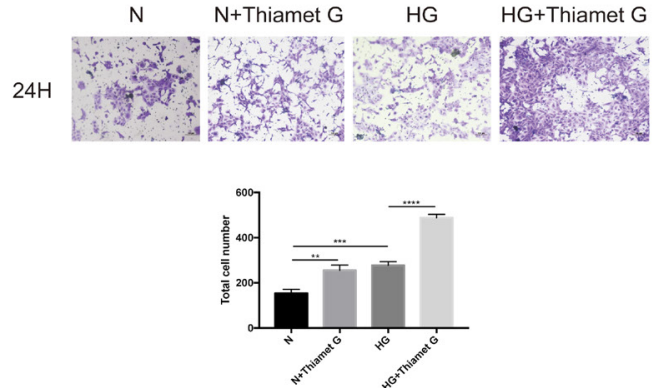

G
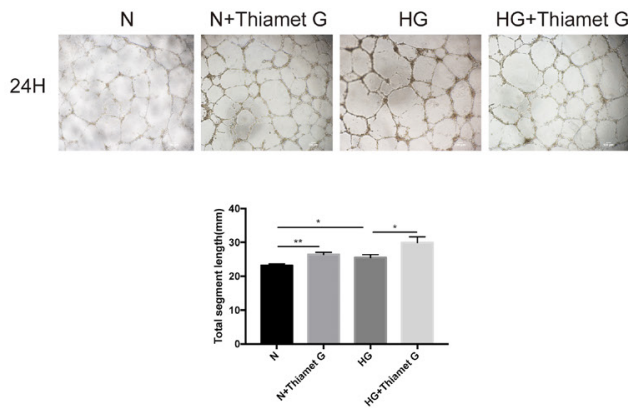

Figure 1 Increased glucose concentration and O-linked $\mathrm{N}$-acetylglucosamine (O-GlcNAc) modification is sufficient for proliferation of human retinal microvascular endothelial cells (HRMECs). Cells were treated with $5.5 \mathrm{mM}$ of glucose (normal (N)) or $5.5 \mathrm{mM}+50 \mu \mathrm{M}$ of Thiamet $\mathrm{G}(\mathrm{N}+$ Thiamet $\mathrm{G}$ ) or $25 \mathrm{mM}$ of glucose (high glucose (HG)) or $25 \mathrm{mM}$ of glucose $+50 \mu \mathrm{M}$ of Thiamet $\mathrm{G}(\mathrm{HG}+$ Thiamet $\mathrm{G})$. (A) The protein lysates were collected and processed by western blot analysis. Western blot analysis was performed using antibodies specific for O-GIcNAc modification and $\beta$-actin (a housekeeping gene). (B) Cell growth was determined by cell counting kit-8 (CCK-8), the graphs present the analysis of cell viability. (C) Cell proliferation was determined by Ki67 staining, the graphs present the analysis of ki67-positive rate. Scale bar $=50 \mu \mathrm{m}$. (D) The apoptosis was detected by western blot analysis. Western blot analysis was performed using antibodies specific for cleaved-caspase 3, Bax, and $\beta$-actin. (E) Scratch-wound assay using HRMECs at 0 (top) and 12 hours (bottom). The black area represents the scratch area. The graphs represent the scratch healing rate. Scale bar $=100 \mu \mathrm{m}$. $(\mathrm{F})$ The migratory ability in HRMECs were detected by Transwell assay. The purple dots represent the cells that completed the vertical migration (passed through the membrane). The graphs represent the numbers of cells that passed through the membrane. Scale bar $=100 \mu \mathrm{m}$. (G) Tube formation ability was detected by tube-forming assay. Cells were re-incubated on Matrigel. The graphs represent total segment length; $n=3$, independent experiments. Scale bar $=100 \mu \mathrm{m}$. ${ }^{*} \mathrm{P}<0.05,{ }^{, * *} \mathrm{p}<0.01,{ }^{* * *} \mathrm{p}<0.001,{ }^{, * * t} \mathrm{p}<0.0001$. 
A

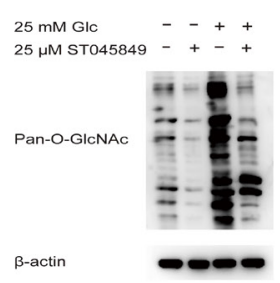

C
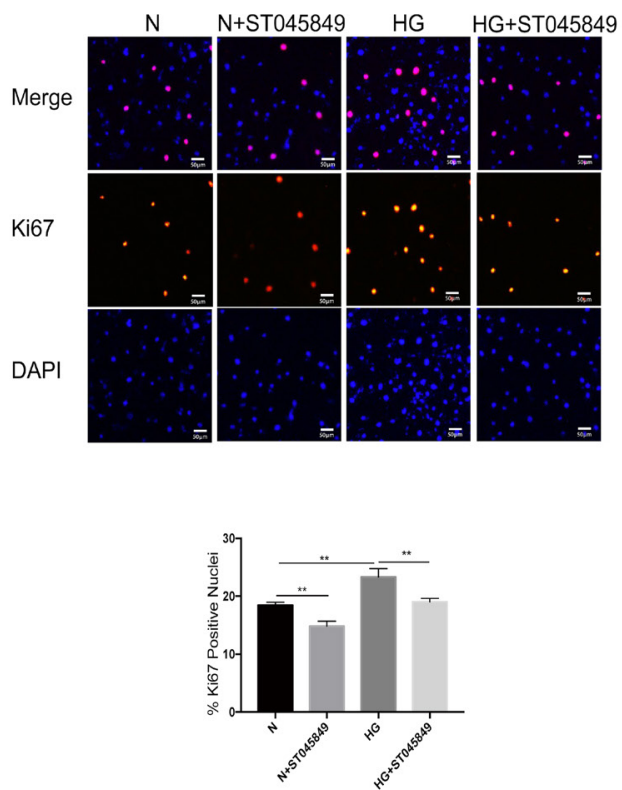

E

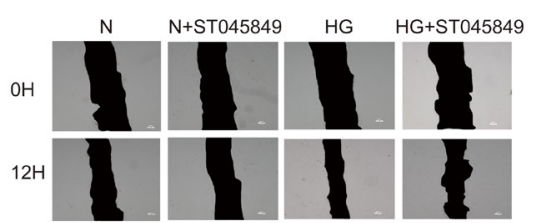

B

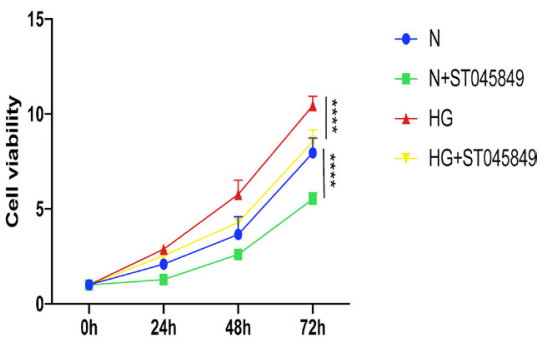

D

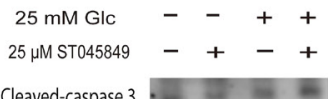

Cleaved-caspase 3

Bax

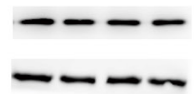

$\mathrm{F}$
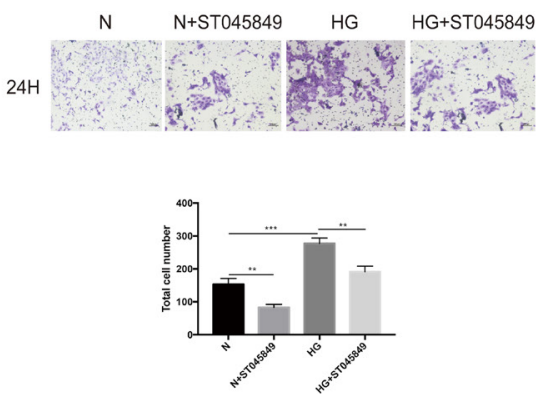

G
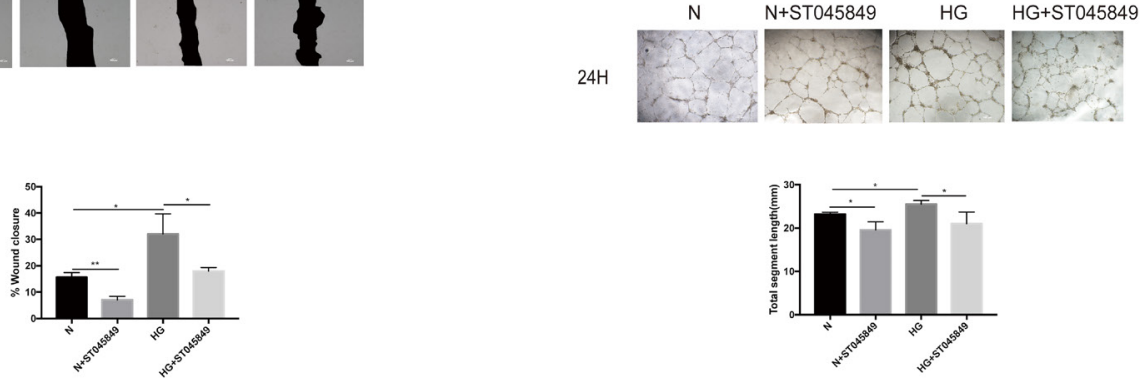

Figure 2 O-linked $\mathrm{N}$-acetylglucosamine (O-GlcNAc) modification is critical for the proliferation and migration of human retinal microvascular endothelial cells (HRMECs). Cells were treated with $5.5 \mathrm{mM}$ of glucose (normal (N)) or $5.5 \mathrm{mM}+25 \mu \mathrm{M}$ of ST045849 (N+ST045849) or $25 \mathrm{mM}$ of glucose (high glucose (HG)) or $25 \mathrm{mM}$ of glucose +25 $\mu \mathrm{M}$ of ST045849 (HG+ST045849). (A) The protein lysates were collected and processed by western blot analysis. Western blot analysis was performed using antibodies specific for O-GlcNAc modification and $\beta$-actin. (B) Cell growth was determined by cell counting kit-8 (CCK-8), the graphs present the analysis of cell viability. (C) Cell proliferation was determined by Ki67 staining, the graphs present the analysis of ki67-positive rate. Scale bar $=50 \mu \mathrm{m}$. (D) The apoptosis was detected by western blot analysis. Western blot analysis was performed using antibodies specific for cleaved-caspase 3, Bax, and $\beta$-actin. (E) Scratch-wound assay using HRMECs at 0 (top) and 12 hours (bottom). The black area represents the scratch area. The graphs represent the scratch healing rate. Scale bar $=100 \mu \mathrm{m}$. (F) The migratory ability in HRMECs were detected by Transwell assay. The purple dots represent the cells that completed the vertical migration (passed through the membrane). The graphs represent the numbers of cells that passed through the membrane. Scale bar $=100 \mu \mathrm{m}$. (G) Tube formation ability was detected by tube-forming assay. Cells were reincubated on Matrigel. The graphs represent total segment length; $n=3$, independent experiments. Scale bar $=100 \mu \mathrm{m}$. ${ }^{*} \mathrm{P}<0.05$, ${ }^{* *} p<0.01,{ }^{* *+*} p<0.001, \quad p<0.0001$. 
A
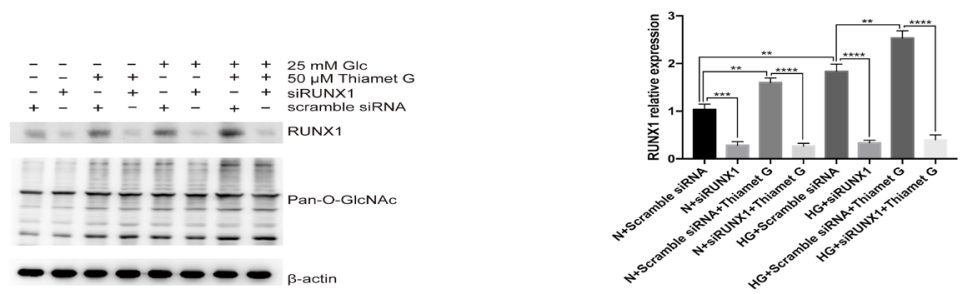

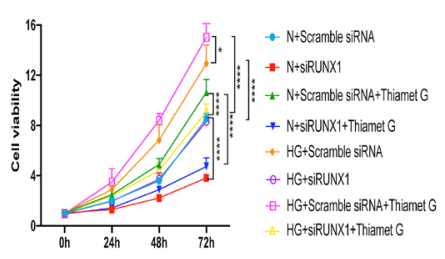

D

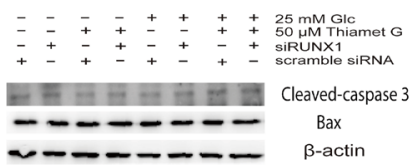

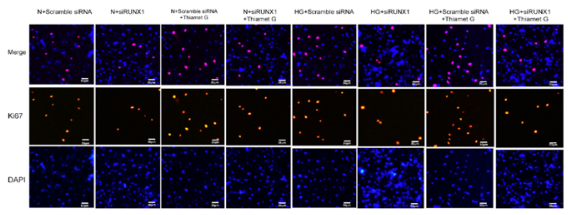
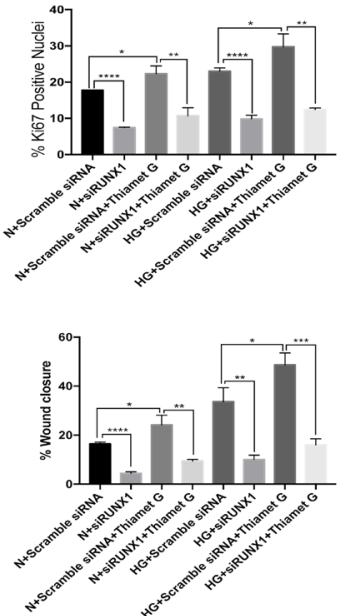

G
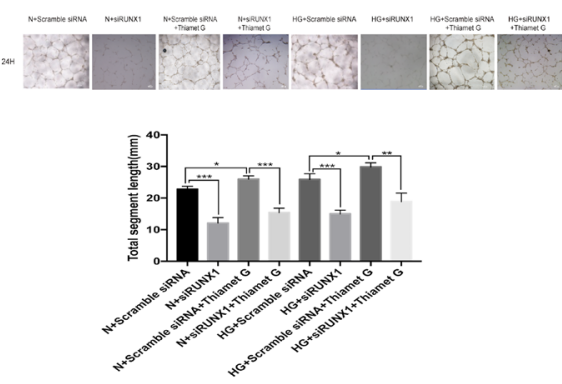

Figure 3 Runt-related transcription factor 1 (RUNX1) mediates the glucose and O-linked N-acetylglucosamine (O-GlcNAc)driven proliferation and migration of human retinal microvascular endothelial cells (HRMECs). Cells were treated with 5.5 $\mathrm{mM}$ of glucose +scramble small interfering RNA (siRNA) (normal (N)+scramble siRNA) or 5.5 mM of glucose +siRUNX1 $(\mathrm{N}+$ siRUNX1) or $5.5 \mathrm{mM}$ of glucose + scramble siRNA $+50 \mu \mathrm{M}$ of Thiamet $\mathrm{G}(\mathrm{N}+$ scramble siRNA+Thiamet $\mathrm{G})$ or $5.5 \mathrm{mM}$ of glucose + siRUNX1+50 $\mu \mathrm{M}$ of Thiamet $\mathrm{G}(\mathrm{N}+\mathrm{siRUNX1+Thiamet} \mathrm{G}$ ) or $25 \mathrm{mM}$ of glucose +scramble siRNA (high glucose $(\mathrm{HG})+$ scramble siRNA) or $25 \mathrm{mM}$ of glucose $+\operatorname{siRUNX1}(\mathrm{HG}+\mathrm{siRUNX1})$ or $25 \mathrm{mM}$ of glucose $+\mathrm{scramble}$ siRNA $+50 \mu M$ of Thiamet $\mathrm{G}(\mathrm{HG}+\mathrm{scramble}$ siRNA+Thiamet $\mathrm{G})$ or $25 \mathrm{mM}$ of glucose +siRUNX1+50 $\mu \mathrm{M}$ of Thiamet G (HG+siRUNX1+Thiamet G). (A) The protein lysates were collected and processed by western blot analysis. Western blot analysis was performed using antibodies specific for O-GlcNAc modification and $\beta$-actin. The graphs present the gray value analysis of RUNX1 band. (B) Cell growth was determined by cell counting kit-8 (CCK-8), the graphs present the analysis of cell viability. (C) Cell proliferation was determined by Ki67 staining, the graphs present the analysis of Ki67-positive rate. Scale bar=50 $\mu$ m. (D) The apoptosis was detected by western blot analysis. Western blot analysis was performed using antibodies specific for cleaved-caspase 3, Bax, and $\beta$-actin. (E) Scratch-wound assay using HRMECs at 0 (top) and 12 hours (bottom). The black area represents the scratch area. The graphs represent the scratch healing rate. Scale bar $=100 \mu \mathrm{m}$. (F) The migratory ability in HRMECs were detected by Transwell assay. The purple dots represent the cells that completed the vertical migration (passed through the membrane). The graphs represent the numbers of cells that passed through the membrane. Scale bar=100 $\mu m$. (G) Tube formation ability was detected by tube-forming assay. Cells were re-incubated on Matrigel. The graphs represent total segment length; $\mathrm{n}=3$, independent experiments. Scale bar $=100 \mu \mathrm{m} .{ }^{\star} \mathrm{P}<0.05,{ }^{\star *} \mathrm{p}<0.01,{ }^{* \star *} \mathrm{p}<0.001,{ }^{\star \star \star *} \mathrm{p}<0.0001$. 

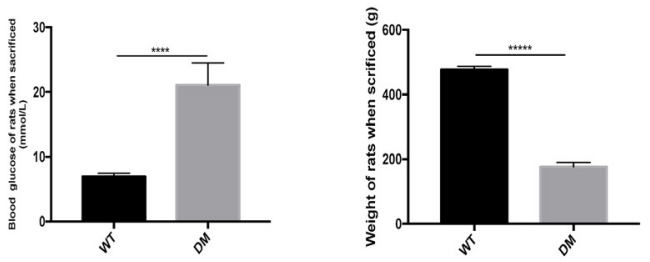

C

IP

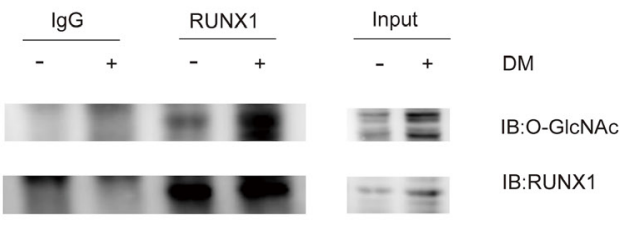

E

IP

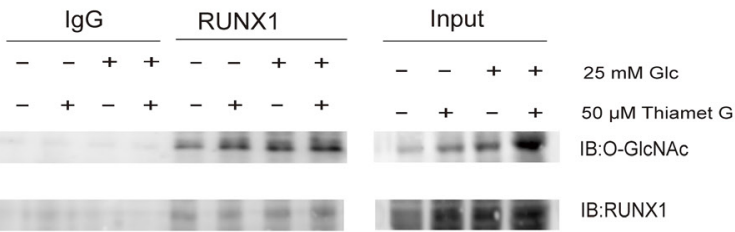

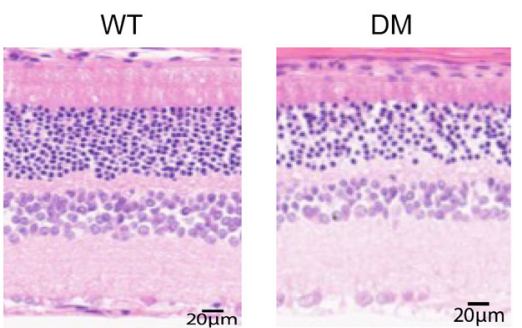

$\mathrm{D}$

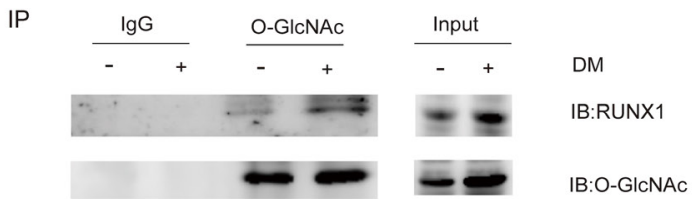

$\mathrm{F}$

IP

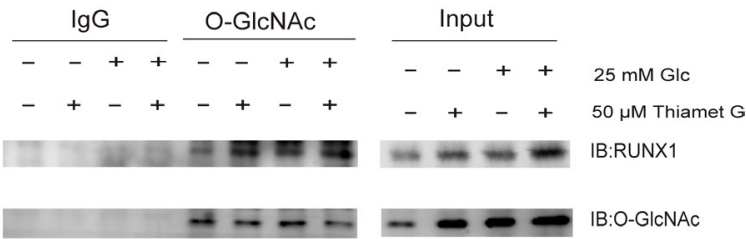

Figure 4 High glucose $(\mathrm{HG})$ environment and treatment of cells with Thiamet $\mathrm{G}$ can promote the O-linked Nacetylglucosamine (O-GlcNAc) modification of Runt-related transcription factor 1 (RUNX1). Rats with diabetes mellitus (DM) were induced by intraperitoneal injection of streptozotocin (STZ). Cells were treated with $5.5 \mathrm{mM}$ of glucose (normal (N)) or $5.5 \mathrm{mM}+50 \mu \mathrm{M}$ of Thiamet $\mathrm{G}(\mathrm{N}+$ Thiamet $\mathrm{G})$ or $25 \mathrm{mM}$ of glucose $(\mathrm{HG})$ or $25 \mathrm{mM}$ of glucose $+50 \mu \mathrm{M}$ of Thiamet G $(\mathrm{HG}+$ Thiamet $\mathrm{G})$ for 48 hours. (A) Blood glucose and body weight of wild-type (WT) and DM rats. (B) H\&E staning of WT and DM rat retina tissues. Scale bar $=20 \mu \mathrm{m}$. (C) O-GlcNAc modification of RUNX1 in retinas was detected by immunoprecipitation (IP), RUNX1, and IgG was immunoprecipitated from the retinas of WT and DM rats (IP), western blot analysis was performed using antibodies specific for O-GIcNAc modification and RUNX1 (IB), IgG, and Input were control groups. (D) O-GIcNAc modification of RUNX1 in retinas was detected by IP, O-GlcNAc, and IgG was immunoprecipitated from the retinas of WT and WT rats (IP), western blot analysis was performed using antibodies specific for O-GlcNAc modification and RUNX1 (IB), IgG, and Input were control groups. (E) O-GIcNAc modification of RUNX1 in HRMECs was detected by IP, RUNX1, and IgG was immunoprecipitated from HRMECs (IP), western blot analysis was performed using antibodies specific for O-GIcNAc modification and RUNX1 (IB), IgG, and Input were control groups (IB), IgG, and Input were control groups. (F) O-GlcNAc modification of RUNX1 in HRMECs was detected by IP, O-GIcNAc, and IgG was immunoprecipitated from HRMECs, western blot analysis was performed using antibodies specific for O-GIcNAc modification and RUNX1 (IB), IgG, and Input were control groups; $n=3$, independent experiments.

RUNX1 can significantly reduce the tube formation. Next, we explored the possible mechanism of RUNX1. STZ is a pancreatic islet $\beta$-cell-cytotoxic antibiotic that can highly and selectively destroy pancreatic islet $\beta$ cells, and induce hypoinsulinemia and hyperglycemia. ${ }^{24}$ STZinduced diabetes in rats is the most commonly used experimental model for investigating DR. ${ }^{25}$ We divided 40 rats into two groups, WT group and STZ-induced DM group, 20 rats in each group. Figure 4A shows the differences of blood glucose and body weight between the two groups. The blood glucose of DM rats is significantly higher than that of WT rats, and the body weight of DM rats is significantly lower than that of WT rats. Before the sacrifice, the retina structure of rats was obviously disordered, which indicated that they had suffered from DR (figure 4B). In order to minimize the effect of individual difference, we mixed the retinal tissues of five rats as a sample, three samples in each group. RUNX1 was immunoprecipitated from samples. The western blot analysis revealed that the RUNX1 protein can be modified by 
O-GlcNAc, and that a hyperglycemic environment can promote its O-GlcNAc modification (figure 4C,D). Then we tested the O-GlcNAc modification status of RUNX1 under $5.5 \mathrm{mM}$ and $25 \mathrm{mM}$ glucose concentrations, with and without Thiamet $\mathrm{G}$, as shown in figure $4 \mathrm{E}, \mathrm{F}$, both $\mathrm{HG}$ condition and treatment of cells with Thiamet G could increase the O-GlcNAc modification of RUNX1.

\section{DISCUSSION}

The findings of this study reveal that hyperglycemia directly affects the function of HRMECs. Furthermore, O-GlcNAcylation is critical for the proliferation and migration of HRMECs, and RUNX1 may take part in this mechanism.

Previous studies have shown that O-GlcNAcylation is involved in the pathological changes of many diseases, such as diabetes, Alzheimer's disease, various cancers, and AIDS. ${ }^{26-28}$ O-GlcNAc modification affects the stability and activity of many transcription factors, such as p53, STAT5, PDX-1, and FOXO1 ${ }^{29-32}$ by modifying these transcription factors and exhibits various effects on diseases. O-GlcNAcylation can reduce the apoptosis of coronary endothelial cells and improve the performance of diabetic heart disease through p53 and histone deacetylase $4 .{ }^{33} 34$ O-GlcNAc modification can also regulate tumor growth and cancer cell proliferation and migration through AMPK, pyruvate kinase M2, and $\beta$-catenin. ${ }^{35-37}$ In DR, the protective effect of increased O-GlcNAc modification against reactive oxygen species has been reported in human retinal endothelial cells. ${ }^{38}$ Overexpression of hyperglycemiainduced intracellular adhesion molecule 1 is linked to the O-GlcNAc modification of transcription factor Sp1 in human umbilical vein endothelial cells and rat retinal capillary endothelial cells. ${ }^{39}$ The increase in O-GlcNAcylation of Sp1 is associated with the upregulated VEGF-A production in the retinal endothelium and pigment epithelium. ${ }^{19}$ These results suggest that O-GlcNAcylation may affect the function of endothelial cells and diabetic neovascularization. The present study provides proof and supplements this conjecture.

Previous studies have shown that RUNX1 targets many signaling pathways, such as transforming growth factor (TGF)- $\beta$ signaling pathway and Wnt signaling pathway. ${ }^{41}$ TGF- $\beta$ can regulate the differentiation, proliferation, apoptosis, and migration of many kinds of cells, and also influence the formation of blood vessels. ${ }^{40}$ The Wnt signaling pathway takes part in cell proliferation, migration, and apoptosis. ${ }^{41}$ Cell proliferation and migration are the processes of angiogenesis. Recent studies have shown that RUNX1 can affect the proliferation and migration of retinal endothelial cells and block endothelial cell tube formation and aberrant angiogenesis in an oxygen-induced retinopathy model. ${ }^{23}$ However, the mechanism has not been demonstrated. In this study, we found that HG could increase RUNX1 expression, this increase was achieved at the transcriptional level because it could not be maintained after siRUNX1 transfection.

Increasing the level of O-GlcNAc modification by treating cells with Thiamet $\mathrm{G}$ can also increase the RUNX1 expression, this increase is ultimately achieved by affecting the transcription level because it could not be maintained after siRUNX1 transfection. Possibly, O-GlcNAc modification can affect other related proteins and thus affect the expression of RUNX1. Whether HG can increase the expression of RUNX1 independently from O-GlcNAcylation is still not clear because HG concentration can increase O-GlcNAc modification by itself, and also can cause many other changes, and the relationship between them is very complex. Recently, it was found that O-GlcNAc can modify RUNX1, and the modification is enhanced in a HG environment. Treatment of cells with Thiamet $G$ also could increase the O-GlcNAcylation. This is consistent with the function of RUNX1 under HG and high O-GlcNAcylation conditions. Therefore, we may suggest that the function of RUNX1 is related to the level of its O-GlcNAc modification.

There are some limitations to the present study. The O-GlcNAc site of RUNX1 was not obtained. Through the specific blocking of the O-GlcNAcylation of RUNX1, its role can be more precisely determined. Furthermore, the impairment of the surrounding cells and microenvironment may have adverse effects on retinal EC as the DR progresses, and this is coupled with both hyperglycemia and increased O-GlcNAcylation. However, further studies on retinal cells and in animal tissues are needed.

In conclusion, the present study reveals that HG condition directly affects retinal EC function, and O-GlcNAcylation is critical for the proliferation and migration of HRMECs. Moreover, RUNX1 may take part in this mechanism, and the function of RUNX1 is related to its O-GlcNAc modification level.

Contributors Conception and design: XX and KL. Data acquisition and analysis: $X X$ and $\mathrm{KL}$. Data interpretation, drafting of the manuscript and approval of the final version: all authors.

Funding This study was supported by National Key R\&D Program of China (grant no: 2019YFC0840607), the National Natural Science Foundation (grant no: 81870667).

Competing interests None declared.

Patient consent for publication Not required.

Ethics approval The experimental protocols used in the present study followed the guidelines established by the ARVO Statement for the Use of Animals in Ophthalmic and Vision Research, and were approved by the Ethics Committee of Shanghai General Hospital, Shanghai Jiaotong University, Shanghai, China.

Provenance and peer review Not commissioned; externally peer reviewed.

Data availability statement All data relevant to the study are included in the article.

Open access This is an open access article distributed in accordance with the Creative Commons Attribution Non Commercial (CC BY-NC 4.0) license, which permits others to distribute, remix, adapt, build upon this work noncommercially, and license their derivative works on different terms, provided the original work is properly cited, appropriate credit is given, any changes made indicated, and the use is non-commercial. See: http://creativecommons.org/ licenses/by-nc/4.0/. 
ORCID iD

Xindan Xing http://orcid.org/0000-0002-7274-8670

\section{REFERENCES}

1 Leasher JL, Bourne RRA, Flaxman SR, et al. Global estimates on the number of people blind or visually impaired by diabetic retinopathy: a meta-analysis from 1990 to 2010. Diabetes Care 2016;39:1643-9.

2 Chung AS, Ferrara N. Developmental and pathological angiogenesis. Annu Rev Cell Dev Biol 2011;27:563-84.

3 Yau JWY, Rogers SL, Kawasaki R, et al. Global prevalence and major risk factors of diabetic retinopathy. Diabetes Care 2012;35:556-64.

4 Gardner TW, Antonetti DA, Barber AJ. Diabetic retinopathy: more than meets the eye. Surv Ophthalmol 2002;47:S253-62.

5 Frank RN. Diabetic retinopathy. N Engl J Med Overseas Ed 2004;350:48-58

6 Semba RD, Huang H, Lutty GA. The role of $O$-GlcNAc signaling in the pathogenesis of diabetic retinopathy. Proteomics Clin Appl 2014;8:218-31.

7 Yokoe S, Asahi M, Takeda T, et al. Inhibition of phospholamban phosphorylation by O-GlcNAcylation: implications for diabetic cardiomyopathy. Glycobiology 2010;20:1217-26.

8 Park M-J, Kim D-I, Lim S-K, et al. High glucose-induced O GlcNAcylated carbohydrate response element-binding protein (ChREBP) mediates mesangial cell lipogenesis and fibrosis: the possible role in the development of diabetic nephropathy. $J$ Biol Chem 2014;289:13519-30.

9 Peterson SB, Hart GW. New insights: A role for $O$-GlcNAcylation in diabetic complications. Crit Rev Biochem Mol Biol 2016;51:150-61.

10 Liu K, Liu H, Zhang Z, et al. The role of $N$-glycosylation in high glucose-induced upregulation of intercellular adhesion molecule-1 on bovine retinal endothelial cells. Acta Ophthalmol 2016;94:353-7.

11 Gurel Z, Zaro BW, Pratt MR, et al. Identification of O-GlcNAc modification targets in mouse retinal pericytes: implication of p53 in pathogenesis of diabetic retinopathy. PLoS One 2014;9:e95561.

12 Aiello LP, Avery RL, Arrigg PG, et al. Vascular endothelial growth factor in ocular fluid of patients with diabetic retinopathy and other retinal disorders. N Engl J Med 1994;331:1480-7.

13 Schröder S, Palinski W, Schmid-Schönbein GW. Activated monocytes and granulocytes, capillary nonperfusion, and neovascularization in diabetic retinopathy. Am J Pathol 1991;139:81-100.

14 Folkman J, Ingber D. Inhibition of angiogenesis. Semin Cancer Biol 1992;3:89-96.

15 Hsu YJ, Hsieh YT, Yeh PT. Combined tractional and rhegmatogenous retinal detachment in proliferative diabetic retinopathy in the antiVEGF era. J Ophthalmol 2014;917375.

16 Kuiper EJ, Van Nieuwenhoven FA, de Smet MD, et al. The angio-fibrotic switch of VEGF and CTGF in proliferative diabetic retinopathy. PLoS One 2008;3:e2675.

17 Saint-Geniez M, Maharaj ASR, Walshe TE, et al. Endogenous VEGF is required for visual function: evidence for a survival role on Mülle cells and photoreceptors. PLoS One 2008;3:e3554.

18 Sang DN, D'Amore PA. Is blockade of vascular endothelial growth factor beneficial for all types of diabetic retinopathy? Diabetologia 2008;51:1570-3.

19 Donovan K, Alekseev O, Qi X, et al. O-GlcNAc modification of transcription factor Sp1 mediates hyperglycemia-induced VEGF-A upregulation in retinal cells. Invest Ophthalmol Vis Sci 2014;55:7862-73

20 Safi SZ, Qvist R, Kumar S. Molecular mechanisms of diabetic retinopathy, general preventive strategies, and novel therapeutic targets. Biomed Res Int 2014;801269.
21 Renneville A, Roumier C, Biggio V, et al. Cooperating gene mutations in acute myeloid leukemia: a review of the literature. Leukemia 2008;22:915-31

22 Miyoshi H, Shimizu K, Kozu T, et al. t(8;21) breakpoints on chromosome 21 in acute myeloid leukemia are clustered within a limited region of a single gene, AML1. Proc Natl Acad Sci U S A 1991;88:10431-4.

23 Lam JD, Oh DJ, Wong LL, et al. Identification of RUNX1 as a mediator of aberrant retinal angiogenesis. Diabetes 2017;66:1950-6.

24 Melmed RN, Benitez CJ, Holt SJ. Intermediate cells of the pancreas. 3. selective autophagy and destruction of beta-granules in intermediate cells of the rat pancreas induced by alloxan and streptozotocin. J Cell Sci 1973;13:297-315.

25 Mazo VK, Sidorova YS, Zorin SN, et al. [Streptozotocin induced diabetes rat models]. Vopr Pitan 2016;85:14-21.

$26 \mathrm{Ma} \mathrm{J}$, Hart GW. Protein O -GlcNAcylation in diabetes and diabetic complications. Expert Rev Proteomics 2013;10:365-80.

27 Jochmann R, Thurau M, Jung S, et al. O-Linked $N$ -Acetylglucosaminylation of Sp1 Inhibits the Human Immunodeficiency Virus Type 1 Promoter. J Virol 2009;83:3704-18.

28 Ma Z. Vosseller K. O-GlcNAc in cancer biology. Amino Acids 2013;45:719-33.

29 Shtraizent N, DeRossi C, Nayar S, et al. MPI depletion enhances O-GlcNAcylation of p53 and suppresses the Warburg effect. Elife 2017;6:e22477.

30 Shin $\mathrm{H}$, Cha H-J, Na K, et al. O -GlcNAcylation of the Tumor Suppressor FOXO3 Triggers Aberrant Cancer Cell Growth. Cancer Res 2018;78:1214-24.

31 Rauth M, Freund P, Orlova A, et al. Cell metabolism control through O-GIcNAcylation of STAT5: a full or empty fuel tank makes a big difference for cancer cell growth and survival. Int J Mol Sci 2019;20:1028.

32 Kebede M, Ferdaoussi M, Mancini A, et al. Glucose activates free fatty acid receptor 1 gene transcription via phosphatidylinositol3-kinase-dependent O-GlcNAcylation of pancreas-duodenum homeobox-1. Proc Natl Acad Sci U S A 2012;109:2376-81.

33 Si R, Zhang Q, Tsuji-Hosokawa A, et al. Overexpression of p53 due to excess protein O-GlcNAcylation is associated with coronary microvascular disease in type 2 diabetes. Cardiovasc Res 2020;116:1186-98.

34 Kronlage M, Dewenter M, Grosso J, et al. O-Glcnacylation of histone deacetylase 4 protects the diabetic heart from failure. Circulation 2019;140:580-94.

35 Kim MY, Kim YS, Kim M, et al. Metformin inhibits cervical cancer cell proliferation via decreased AMPK O-GIcNAcylation. Animal Cells Syst 2019;23:302-9.

36 Singh JP, Qian K, Lee J-S, et al. O-GlcNAcase targets pyruvate kinase M2 to regulate tumor growth. Oncogene 2020;39:560-73.

37 Gao S, Miao Y, Liu Y, et al. Reciprocal regulation between OGlcNAcylation and $\beta$-catenin facilitates cell viability and inhibits apoptosis in liver cancer. DNA Cell Biol 2019;38:286-96.

38 Liu GUOD, Xu C, Feng LE, et al. The augmentation of OGlcNAcylation reduces glyoxal-induced cell injury by attenuating oxidative stress in human retinal microvascular endothelial cells. Int $J$ Mol Med 2015;36:1019-27.

39 Zhang Y, Qu Y, Niu T, et al. O -GlcNAc modification of Sp1 mediates hyperglycaemia-induced ICAM-1 up-regulation in endothelial cells. Biochem Biophys Res Commun 2017;484:79-84.

40 Miyazono K, Suzuki H, Imamura T. Regulation of TGF-beta signaling and its roles in progression of tumors. Cancer Sci 2003;94:230-4.

41 Naillat F, Yan W, Karjalainen R, et al. Identification of the genes regulated by Wnt-4, a critical signal for commitment of the ovary. Exp Cell Res 2015;332:163-78. 